${ }^{1}$ Department of Surgical Oncology, Copernicus Provincial Multidisciplinary Centre of Oncology and Traumatology, Paderewskiego, Lodz, Poland 2Department of Head and Neck Cancer Surgery, Cancer Center, Medical University of Lodz, Paderewskiego, Lodz, Poland

\title{
Liquid biopsy in targeting gene polymorphism related to the response within immunocheckpoint inhibitors therapeutic regimen
}

\author{
Corresponding author: \\ Katarzyna Boguszewska-Byczkiewicz, \\ Department of Surgical Oncology, \\ Copernicus Provincial Multidisciplinary \\ Centre of Oncology and Traumatology, \\ Paderewskiego 4 Str. \\ 93-510 Lodz, Poland \\ e-mail: \\ kkatarzynabboguszewska@gmail.com
}

Medical Research Journal 2021

Volume 6, Number 3, 245-248

10.5603/MRJ.a2021.0022

Copyright (C) 2021 Via Medica

ISSN 2451-2591

e-ISSN 2451-4101

\begin{abstract}
Immunotherapy belongs to the group of targeted therapies; it is based on natural immune mechanisms which axis can be promoted or blocked at appropriate points. Breast cancer is the world's most common cancer among women and in March 2019 the FDA approved the first immunopharmaceutical Atezolizum$\mathrm{ab}$, for the treatment of breast cancer. So far, the only registered marker for classification for checkpoint inhibitor therapy has been the presence of PD-L1 receptor expression in tumour cells.

A comprehensive search of the literature to elucidate the correlation between PD-1/PD-L1 single nucleotide polymorphism (SNP) and cancer, especially breast cancer or other diseases susceptibility and PD-1/ PD-L1 expression.

Seven susceptibility loci was considered: rs41386349, rs7421861, rs36084323, rs11568821, rs2227981, rs10204525, rs2227982. Three of them may be taken into account as potentially helpful in breast cancer patient treatment tailoring: rs36084323, rs2227981, rs2227982.

Key words: PD-1, PD-L1, immunocheckpoint inhibition, immunogenetics, single nucleotide polymorphism, breast cancer, liquid biopsy
\end{abstract}

Med Res J 2021; 6 (3): 245-248

\section{Introduction}

Liquid biopsy (LB) is the most superior diagnostic method for the determination of the tumour signature in blood [1].

Breast cancer is the most common female cancer worldwide. Only in 2018, it reached the top rate among 25 cancers for 40 countries in Europe $(523,000$ cases, within 3.91 million new cases of cancer per year in both genders) [2]. In simplified words, the prognosis depends on the tumour type (lumina, basal-like, triple-negative) and its stage (histological grade, axillary lymph node involvement and distant metastasis) at the time of diagnosis. Despite low immunogenicity in breast cancer [3], there is a rising interest in anti-programmed dead receptor 1 and programmed dead ligand 1 (anti-PD1/anti-PD-L1) treatment in a special subset of triple-negative breast cancer (TNBC) and human epidermal growth factor receptor 2 positive (HER-2 positive) [4-6]. In March 2019, FDA approved Atezolimumab (Tecentriq) for adult patients with unresectable, locally advanced or metastatic triple-negative breast cancer (TNBC) the tumours of which express PD-L1 as determined by an FDA-approved test [7].

PD-1 firstly identified by Ishida in 1992 [8] is a transmembrane glycoprotein located in tumour cells, cytotoxic T-lymphocytes, natural killer (NK), B-lymphocytes, monocytes and other tissues infiltrating lymphocytes (TILs) [9]. PD-1 receptors linked with their ligands PD-L1 identified in 1999 [10] on an antigen presenting cell (APC) inhibit antitumour activity. PD-1/ PD-L1 axis block activation of cytotoxic T-cell. This blockade plays a critical role in tumour resistance mechanisms. The suspension of PD-1/PD-L1 axis can restore T-cell and promote immunity against tumour. Antitumour effect exerted via immunocheckpoint inhibitors (Atezolizumab, 
Avelumab, Nivolumab, Pembrolizumab) is based on the promotion of a cell-mediated immune response. To date, for the purpose of pretreatment qualification, FDA-approved only the PD-L1 expression level, as determined by an IHC based on the VENTANA assai [7] for a primary or metastatic tissue source. It is still unclear if PD-1(PDCD1)/PD-L1(Pdcd1I2) gene single nucleotide polymorphism (SNP) could be a predictor for immunocheckpoint inhibition treatment, especially in the context of breast cancer risk and prognosis [11, 12]. The frequencies of rs36084323 GG genotype and rs2227981 CT genotype can affect the susceptibility of breast cancer [12]. The human gene encoding PD-1 is located on $2 q 37.3$ [12].

\section{Programmed death-1 (costimulatory molecule, PD-1, CD279) gene polymorphism/ PDCD1 (coding for programmed cell death-1)}

Loci of $P D$-1 gene might be a potential biomarker for predicting susceptibility to therapeutic markers for cancer treatment. However, the identification of biomarkers able to predict a clinical benefit of PD-1/PD-L1 inhibitors seems to be a challenge. To apply liquid biopsy into minimal invasive stratification, single nucleotide polymorphism (SNP) should be designated. Seven susceptibility loci for immunotherapy effect in breast cancer patients can be considered: rs41386349, PDCD1 (rs7421861), PD-1.1 (rs36084323), PDCD-1.3 (rs11568821), PDCD-1.5 (rs2227981), PDCD-1.6(rs10204525), PDCD-1.9 (rs2227982). Previous studies have discussed methods and combined biomarkers from different points of view: tumour mutation and neoantigens burden as well as some oncogene mutations like EGFR, ALK, KRAS and STK11 [13].

However, PD-L1 expression measured using the $\mathrm{IHC}$ is the only FDA-approved test. Lower PD-1 expression has been found to be associated with $G$ of rs10204525 [14].

\section{Certain single nucleotide polymorphisms (SNPs) in genes such as PDCD1 (coding for programmed cell death)}

Rs41386349, this SNP was previously considered as a hot point for Grave's disease and Addison's disease [15], rheumatoid arthritis (RA) [16], Kawasaki disease [17], multiple myeloma [18] and variant A for rs41386349 affects susceptibility of chronic HBV [19]. Whereas, only PD-L1 rs1411262C/T gene polymorphism in the case of Grave's disease and Addison's disease [15], increased rheumatoid factor seropositivity [16] and a higher probability of Kawasaki disease [17] was discovered. The multiple myeloma occurs more often with a PDCD1 GCC/GCC haplotype (rs36084323/rs41386349/rs2227982) variant [18].

PDCD1 (rs7421861) has been studied in case of esophageal cancer [20] and chronic HBV infection, its oncogenicity [19] as well as RA [16]. PDCD1 gene polymorphisms influence the severity of a disease and are associated with distant metastasis, higher TNM stage, higher PD-1 gene and plasma levels in esophageal cancer patients and risk of esophageal cancer in general [20]. This gene variability may play a role in hepatocarcinogenesis caused by chronic HBV infection [19]. Tseng $\mathrm{CC}$ et al. have found higher expression of PDCD1 in RA compared to controls. However, PDCD1 gene polymorphisms were similar in this study [16].

PD-1.1 (rs36084323) has been considered in a case of breast cancer [21], epithelial ovarian cancer [22] esophageal cancer [20], NSCLC [23], overall cancer risk [24], RA [16] pregnancy losses [25] and aplastic anemia [26]. Therefore, significantly lower frequencies of PD-1.1 GG have been presented in women with breast cancer [21]. Rs36084323 polymorphism predicts epithelial ovarian cancer development [22]. No association with esophageal cancer metastasis [20], no differences in the distribution of the PD-1.1 alleles in NSCLC [23], no association between overall cancer risk have been obtained [24]. For autoimmune diseases, such as RA, rs36084323 decreased an inadequate response to conventional synthetic disease-modifying antirheumatic drugs [16]. Hayashi Y. et al. have evaluated the association of genetic variants of PD1 with recurrent pregnancy loss with significantly higher frequencies of rs36084323 in women with two or more pregnancy losses [25]. Polymorphism of PD1.1 has been rare in the case of patients with aplastic anaemia [26].

PDCD-1.3 (rs11568821) gene polymorphism has been considered to be related to an overall cancer risk [24, 27] and SLE [28]. Researchers have found a decreased overall cancer risk in case of PDCD-1.3, variant TC [24, 27]. PD1.3GG genotype and $G$ allele have been significantly more frequent in SLE patients [28]. Prokunina et al. have found that this genetic variant would affect PD-1 mRNA level by changing the binding affinity of RUNX (a transcriptional factor of PD-1) [29].

PDCD-1.5 (rs2227981) variant TT has been associated with a decreased risk of cancer [24, 27]. CT genotype has been significantly lower in breast cancer women [21], whereas an increase in the risk of cervical [30], T-allele of rs2227981 gene polymorphism reduced risk of epithelial ovarian cancer [22], lung [31], gastric [32], colon [33], thyroid cancers [34] and CC variant was significantly more frequent in patients suffering from SLE [28]. 
PDCD-1.6 (rs10204525) has been explored with respect to the associations between $P D-1.6$ gene polymorphisms and esophageal cancer [20,35], overall cancer risk [24], aplastic anemia [26], juvenile idiopathic arthritis [36] and HBV infection [37]. GG genotype of rs10204525 polymorphism has increased the risk of esophageal cancer in contrast to the more common AA genotype which was associated with distant metastasis and higher PD-1 gene and plasma levels $[20,35]$. Even in some other work, there is no relation between this SNP and overall cancer risk [24]. Furthermore, some variants of rs10204525 have been linked to aplastic anaemia [26], while the CT variant was associated with juvenile idiopathic arthritis and linked to Anti-CCP antibodies, RF, and the CHAQ score [36]. Next, subjects carrying minor allele $G$ had a significantly decreased risk of getting infected with HBV and were associated with lower PD-1 expression [14], variant AA of PD-1 rs10204525 was prone to higher PD-1 expressions in tumour tissues, peri-tumour tissues and cirrhotic tissues [37]. GG genotype variant altered tumour necrosis factor- $\alpha$ (TNF- $\alpha$ ) to increase levels in HBV patients [38].

PDCD-1.9 (rs2227982) has been evaluated in terms of breast cancer [39], ovarian cancer [40], ankylosing spondylitis [41], multiple myeloma [18] and chronic HBV infection and its oncogenity[19]. This polymorphism increases the probability and severity of the disease in case of ankylosing spondylitis and breast cancer $[39,41]$. Simultaneously, the same reserchers have found that $\mathrm{C}>\mathrm{T}$ variant reduces the risk of breast cancer [39] and raises the risk of ovarian cancer [40]. CC genotype variant was significantly correlated with a higher frequency of osteolysis [18]. This gene polymorphism may have a significant influence on hepatocarcinogenesis [19]. However, CC of rs2227982 variant had a shielding role in HBV infection [14].

\section{Conclusions}

In summary, there are no reports on PD-1 gene polymorphism and its association with immunotherapy favourable outcome susceptibility. Seven susceptibility loci was considered: rs41386349, rs7421861, rs36084323, rs11568821, rs2227981, rs10204525, rs2227982. The important question for developing next-generation anti-PD-1/PD-L1 antibodies is whether the therapeutic effect can be predicted with SNP analysis using liquid biopsy. Nevertheless, three SNP may be taken into account in the breast cancer patient: rs36084323, rs2227981, rs2227982.

Conflict of interest: The authors have no conflicts of interest to declare regarding this study.

\section{References}

1. Speicher MR, Pantel K. Tumor signatures in the blood. Nat Biotechnol. 2014; 32(5): 441-443, doi: 10.1038/nbt.2897, indexed in Pubmed: 24811515.

2. Ferlay J, Colombet M, Soerjomataram I, et al. Cancer incidence and mortality patterns in Europe: Estimates for 40 countries and 25 major cancers in 2018. Eur J Cancer. 2018; 103: 356-387, doi: 10.1016/j. ejca.2018.07.005, indexed in Pubmed: 30100160.

3. Yarchoan M, Hopkins A, Jaffee EM. Tumor mutational burden and response rate to PD-1 inhibition. N Engl J Med. 2017; 377(25): 25002501, doi: 10.1056/NEJMc1713444, indexed in Pubmed: 29262275.

4. Vikas P, Borcherding N, Zhang $\mathrm{W}$. The clinical promise of immunotherapy in triple-negative breast cancer. Cancer Manag Res. 2018; 10: 6823-6833, doi: 10.2147/CMAR.S185176, indexed in Pubmed: 30573992.

5. Adams S, Schmid P, Rugo HS, et al. Pembrolizumab monotherapy for previously treated metastatic triple-negative breast cancer: cohort A of the phase II KEYNOTE-086 study. Ann Oncol. 2019; 30(3): 397-404, doi: 10.1093/annonc/mdy517, indexed in Pubmed: 30475950.

6. Adams S, Loi S, Toppmeyer D, et al. Pembrolizumab monotherapy for previously untreated, PD-L1-positive, metastatic triple-negative breast cancer: cohort B of the phase II KEYNOTE-086 study. Ann Oncol. 2019; 30(3): 405-411, doi: 10.1093/annonc/mdy518, indexed in Pubmed: 30475947.

7. FDA approves atezolizumab for PD-L1 positive unresectable locally advanced or metastatic triple-negative breast cancer. Case Medical Research. 2019. https://www.fda.gov/drugs/drug-approvals-and-databases/fda-approves-atezolizumab-pd-11-positive-unresectable-loc ally-advanced-or-metastatic-triple-negative.

8. Ishida $Y$, Agata $Y$, Shibahara K, et al. Induced expression of PD-1, a novel member of the immunoglobulin gene superfamily, upon programmed cell death. The EMBO Journal. 1992; 11(11): 3887-3895, doi: 10.1002/j.1460-2075.1992.tb05481.x, indexed in Pubmed: 1396582.

9. Sharpe $A H$, Wherry EJ, Ahmed R, et al. The function of programmed cell death 1 and its ligands in regulating autoimmunity and infection. Nat Immunol. 2007; 8(3): 239-245, doi: 10.1038/ni1443, indexed in Pubmed: 17304234 .

10. Dong H, Zhu G, Tamada K, et al. B7-H1, a third member of the B7 family, co-stimulates T-cell proliferation and interleukin-10 secretion. Nat Med. 1999; 5(12): 1365-1369, doi: 10.1038/70932, indexed in Pubmed: 10581077

11. Ren HT, Li YM, Wang XJ, et al. PD-1 rs2227982 Polymorphism Is Associated With the Decreased Risk of Breast Cancer in Northwest Chinese Women: A Hospital-Based Observational Study. Medicine (Baltimore). 2016; 95(21): e3760, doi: 10.1097/MD.0000000000003760, indexed in Pubmed: 27227944.

12. Hua Z, Li D, Xiang G, et al. PD-1 polymorphisms are associated with sporadic breast cancer in Chinese Han population of Northeast China Breast Cancer Res Treat. 2011; 129(1): 195-201, doi: 10.1007/s10549011-1440-3, indexed in Pubmed: 21487727.

13. Teng F, Meng $X$, Kong Li, et al. Progress and challenges of predictive biomarkers of anti PD-1/PD-L1 immunotherapy: A systematic review. Cancer Lett. 2018; 414: 166-173, doi: 10.1016/j.canlet.2017.11.014, indexed in Pubmed: 29155348.

14. Huang C, Ge T, Xia C, et al. Association of rs 10204525 genotype GG and rs2227982 CC combination in programmed cell death 1 with hepatitis B virus infection risk. Medicine (Baltimore). 2019; 98(35): e16972, doi: 10.1097/MD.0000000000016972, indexed in Pubmed: 31464942.

15. Mitchell $A$, Cordell $H$, Soemedi $R$, et al. Programmed death ligand 1 (PD-L1) gene variants contribute to autoimmune addison's disease and graves' disease susceptibility. Molecular Endocrinology. 2009; 23(11): 1935-1935, doi: 10.1210/mend.23.11.9997, indexed in Pubmed: PMC5419164.

16. Tseng CC, Lin YZ, Lin CH, et al. Genetic and epigenetic alteration of the programmed cell death 1 in rheumatoid arthritis. Eur J Clin Invest. 2019; 49(10): e13094, doi: 10.1111/eci.13094, indexed in Pubmed: 30810221.

17. Chun JK, Kang DW, Yoo BW, et al. Programmed death-1 (PD-1) gene polymorphisms lodged in the genetic predispositions of Kawasaki Disease. Eur J Pediatr. 2010; 169(2): 181-185, doi: 10.1007/s00431009-1002-4, indexed in Pubmed: 19468750

18. Kasamatsu T, Awata M, Ishihara R, et al. PDCD1 and PDCD1LG1 polymorphisms affect the susceptibility to multiple myeloma. Clin Exp Med. 2020; 20(1): 51-62, doi: 10.1007/s10238-019-00585-4, indexed in Pubmed: 31620907 
19. Li F, Fan X, Wang X, et al. Genetic association and interaction of PD1 and TIM3 polymorphisms in susceptibility of chronic hepatitis $B$ virus infection and hepatocarcinogenesis. Discov Med. 2019; 27(147) 79-92, indexed in Pubmed: 30939292.

20. Zang B, Chen C, Zhao JQ. PD-1 gene rs10204525 and rs7421861 polymorphisms are associated with increased risk and clinical features of esophageal cancer in a Chinese Han population. Aging (Albany NY). 2020; 12(4): 3771-3790, doi: 10.18632/aging.102845, indexed in Pubmed: 32084010

21. Hua Z, Li D, Xiang G, et al. PD-1 polymorphisms are associated with sporadic breast cancer in Chinese Han population of Northeast China. Breast Cancer Res Treat. 2011; 129(1): 195-201, doi: 10.1007/s10549011-1440-3, indexed in Pubmed: 21487727.

22. Li Y, Zhang HL, Kang S, et al. The effect of polymorphisms in PD-1 gene on the risk of epithelial ovarian cancer and patients' outcomes. Gynecol Oncol. 2017; 144(1): 140-145, doi: 10.1016/j.ygyno.2016.11.010, indexed in Pubmed: 27836206.

23. Ma $Y$, Liu $X$, Zhu J, et al. Polymorphisms of co-inhibitory molecules (CTLA-4/PD-1/PD-L1) and the risk of non-small cell lung cancer in a Chinese population. 8(9):16585-91, 2015. Int J Clin Exp Med. 2015 8(9): 16585-16591, indexed in Pubmed: 26629188.

24. Hashemi M, Karami S, Sarabandi S, et al. Association between and Polymorphisms and the Risk of Cancer: A Meta-Analysis of Case-Contro Studies. Cancers (Basel). 2019; 11(8), doi: 10.3390/cancers11081150, indexed in Pubmed: 31405171.

25. Hayashi Y, Nishiyama T, Nakatochi M, et al. Association of genetic variants of with recurrent pregnancy loss. Reprod Med Biol. 2018; 17(2): 195-202, doi: 10.1002/rmb2.12093, indexed in Pubmed 29692678

26. WuZ, Miao M, Qiu Y, etal. Association between polymorphisms in PDCD1 gene and aplastic anemia in Chinese Han population. Leuk Lymphoma. 2013; 54(10): 2251-2254, doi: 10.3109/10428194.2013.772605 indexed in Pubmed: 23373967.

27. Zhang J, Zhao T, Xu C, et al. The association between polymorphisms in the PDCD1 gene and the risk of cancer: A PRISMA-complian meta-analysis. Medicine (Baltimore). 2016; 95(40): e4423, doi: 10.1097/MD 0000000000004423, indexed in Pubmed: 27749524.

28. Abo El-Khair SM, Sameer W, Awadallah N, et al. Programmed cell death 1 gene polymorphism as a possible risk for systemic lupus erythematosus in Egyptian females. Lupus. 2019; 28(12): 1427-1434, doi: 10.1177/0961203319878493, indexed in Pubmed: 31551030

29. Prokunina L, Castillejo-López C, Oberg F, et al. A regulatory polymorphism in PDCD1 is associated with susceptibility to systemic lupus erythematosus in humans. Nat Genet. 2002; 32(4): 666-669, doi 10.1038/ng1020, indexed in Pubmed: 12402038.

30. Li XF, Jiang XQ, Zhang JW, et al. Association of the programmed cell death-1 PD1.5 C > T polymorphism with cervical cancer risk in a Chinese population. Genet Mol Res. 2016; 15(1), doi: 10.4238/gmr.15016357. indexed in Pubmed: 27050970.

31. Yin L, Guo H, Zhao L, et al. The programmed death-1 gene polymorphism (PD-1.5 C/T) is associated with non-small cell lung cancer risk in a Chinese Han population. . Int J Clin Exp Med. 2014; 7(12): 5832-5836, indexed in Pubmed: 25664115

32. Savabkar S, Azimzadeh P, Chaleshi V, et al. Programmed death-1 gene polymorphism (PD-1.5 C/T) is associated with gastric cancer. Gastroenterol Hepatol Bed Bench. 2013; 6(4): 178-182, indexed in Pubmed: 24834269.

33. Mojtahedi Z Mohmedi M Rahimifar S et al Programmed death-1 gene polymorphism (PD-1.5 C/T) is associated with colon cancer. Gene. 2012; 508(2): 229-232, doi: 10.1016/j.gene.2012.07.059, indexed in Pubmed: 22892379

34. Haghshenas MR, Dabbaghmanesh MH, Miri A, et al. Association of PDCD1 gene markers with susceptibility to thyroid cancer. J Endocrinol Invest. 2017; 40(5): 481-486, doi: 10.1007/s40618-016-0579-5, indexed in Pubmed: 27943063

35. Qiu H, Zheng L, Tang W, et al. Programmed death-1 (PD-1) polymorphisms in Chinese patients with esophageal cancer. Clin Biochem. 2014; 47(7-8): 612-617, doi: 10.1016/j.clinbiochem.2013.12.023, indexed in Pubmed: 24389076

36. Ali MA, Abdelaziz A, Ali M, et al. PADI4 (rs2240340), PDCD1 (rs10204525), and CTLA4 (231775) gene polymorphisms and polyarticular juvenile idiopathic arthritis. Br J Biomed Sci. 2020; 77(3): 123-128, doi: 10.1080/09674845.2020.1730626, indexed in Pubmed: 32163016.

37. Li Z Li Na, Li F et al. Immune checkpoint proteins PD-1 and TIM-3 are both highly expressed in liver tissues and correlate with their gene polymorphisms in patients with HBV-related hepatocellular carcinoma. Medicine (Baltimore). 2016; 95(52): e5749, doi: 10.1097/MD.0000000000005749, indexed in Pubmed: 28033288.

38. Zhang G, Li Z, Han Q, et al. Altered TNF- $\alpha$ and IFN- levels associated with PD1 but not TNFA polymorphisms in patients with chronic HBV infection. Infect Genet Evol. 2011; 11(7): 1624-1630, doi: 10.1016/j. meegid.2011.06.004, indexed in Pubmed: 21712100

39. Ren HT, Li YM, Wang XJ, et al. PD-1 rs2227982 polymorphism is associated with the decreased risk of breast cancer in northwest Chinese women: a hospital-based observational study. Medicine (Baltimore). 2016; 95(21): e3760, doi: 10.1097/MD.0000000000003760, indexed in Pubmed: 27227944

40. Tan D, Sheng Li, Yi QH. Correlation of PD-1/PD-L1 polymorphisms and expressions with clinicopathologic features and prognosis of ovarian cancer. Cancer Biomark. 2018; 21 (2): 287-297, doi: 10.3233/CBM170357, indexed in Pubmed: 29171986.

41. Chen Si, Li Y, Deng C, et al. The associations between PD-1, CTLA-4 gene polymorphisms and susceptibility to ankylosing spondylitis: a meta-analysis and systemic review. Rheumatol Int. 2016; 36(1): 33-44, doi: 10.1007/s00296-015-3327-9, indexed in Pubmed: 26232179 